\title{
Desenvolvimento de protótipos de hardware com baixo custo para inclusão digital de pessoas com deficiência motora
}

\author{
Isadora R F Muniz ${ }^{1}$, Denise Stringhini - Orientadora ${ }^{1}$ \\ ${ }^{1}$ Instituto de Ciência e Tecnologia - Universidade Federal de São Paulo (UNIFESP) \\ São José dos Campos - SP - Brazil \\ \{isadora.muniz, dstringhini\}@unifesp.br
}

\begin{abstract}
This project aims to help people with motor and speech disabilities to use the computer. The results of studies and their applications for the development of a low-cost prototype will be presented in this work. Different methods for capturing and interpreting an external signal have been developed and tested, which make it possible to send the signal from an external device to the computer in order to control it with just a touch or movement. The interaction with the computer is performed through an existing interpreter software integrated with the developed prototypes. To test its performance the participation of a volunteer was obtained, which was an adult with motor problems resulting from cerebral palsy.
\end{abstract}

Resumo. O objetivo do presente trabalho é auxiliar pessoas com deficiência motora e de fala a utilizarem o computador. Serão apresentados os estudos $e$ aplicações feitos para o desenvolvimento de um protótipo de baixo custo. Foram desenvolvidos e testados diferentes métodos para captura e interpretação de sinal que possibilitam o seu envio de um dispositivo externo para o computador de forma a controlá-lo com apenas um toque ou movimento. A interação com o computador é realizada através de um software interpretador integrado aos protótipos desenvolvidos. Para a realização dos testes, obteve-se a participação de um voluntário adulto possuidor de problemas motores decorrentes de paralisia cerebral.

\section{Introdução}

A falta de acessibilidade em equipamentos de tecnologia é notória, embora perceba-se um aumento de esforços neste sentido. Com o passar dos anos, novos computadores, celulares e tablets são lançados, porém, suas tecnologias não têm acessibilidade suficiente para aqueles usuários que possuem deficiência específica e acentuada na coordenação motora e de fala, impossibilitando que usufruam de seus benefícios.

Apesar de existirem dispositivos - que são poucos no mercado - desenvolvidos com o intuito de trazer acesso às pessoas com deficiência motora acentuada, o preço a ser pago normalmente é alto, sendo este mais um obstáculo encontrado. Pensando em tornar acessível tanto para a usabilidade quanto para o custo, a intenção deste projeto desde o seu início é o desenvolvimento de um dispositivo que seja de baixo custo e que supra as necessidades destes usuários.

Como primeiros passos do projeto, obteve-se seu embasamento através da busca por informações referentes às tecnologias de rastreamento ocular - também conhecida 
como eye tracking. Porém, esta foi descartada por dois motivos. O primeiro, é o fato desta tecnologia normalmente possuir um elevado custo, com dispositivos custando em média $\mathrm{R} \$ 1000,00$. O segundo, é relacionado ao fato de que o usuário voluntário não teve boa adaptação a este tipo de tecnologia, pois não possui a firmeza necessária na região do pescoço. Assim, procurou-se por tecnologias alternativas, como sensores de movimento que, com o mínimo de movimento do usuário, possibilite a realização de alguma função.

Outro ponto importante para o desenvolvimento deste tipo de protótipo é a necessidade de sua integração a algum software de interpretação. Por meio de pesquisas, foram encontrados softwares com funcionamento de diversas formas, seja pelo processamento de imagem captada por webcam ou através de comando realizado pela ponteira do mouse. Diversos testes foram realizados com um usuário voluntário, obtendo-se maior benefício a partir daqueles baseados em um comando dado por um botão externo de teclado do computador, cujo desenvolvimento e funcionamento será explicado de forma detalhada nas seções a seguir.

\title{
2. Revisão da Literatura
}

Para a realização do projeto, buscou-se entender a situação do usuário alvo, quais as possíveis causas que acarretam em sua condição de deficiência, se para os diferentes traumas as sequelas adquiridas se distinguem, analisando como e porque isto ocorre.

\subsection{Deficiência Física}

Segundo [Macedo 2008], tem-se a definição do conceito de deficiência motora (ou deficiência física) como:

\begin{abstract}
"As chamadas deficiências físicas congênitas definem-se como qualquer perda ou anormalidade de estrutura ou função fisiológica ou anatômica, desde o nascimento, decorrente de causas variadas, como por exemplo: prematuridade, anóxia perinatal, desnutrição materna, rubéola, toxoplasmose, trauma de parto, exposição à radiação, uso de drogas, causas metabólicas e outras desconhecidas."
\end{abstract}

Somado às variadas causas da deficiência citadas pelo mesmo trabalho, incluemse doenças degenerativas, como a Esclerose Lateral Amiotrófica (ELA), além de Paralisia Cerebral.

Sendo assim, a condição de deficiência está associada a diversos contextos, ocorrendo por múltiplas causas, múltiplos tipos clínicos, múltiplas associações de desenvolvimento de patologias, assim como múltiplos padrões de neuropatologias no mapeamento do cérebro.

Logo, segundo [MacLennan et al. 2015], "essas diferentes causas e etiologias cada um têm um resultado em uma desordem não-específica e não-progressiva da postura e controle de movimento". Ou seja, para cada trauma há uma sequela resultante diferente o que fez com que inicialmente o projeto buscasse um usuário específico com graves sequelas de paralisia cerebral. 


\subsection{Tecnologia Assistiva}

O termo Tecnologia Assistiva se refere a criações voltadas ao auxílio de pessoas com deficiência, tornando sua vida mais fácil e independente, proporcionando melhor qualidade de vida e inclusão social, agindo de forma a ampliar a mobilidade, comunicação e habilidades de aprendizado. [Bersch 2013]

Com o intuito de compreender como as tecnologias já existentes funcionam e obter a base de uma possível forma de funcionamento do protótipo para desenvolvimento, pesquisou-se por tecnologias que têm se destacado ao longo dos últimos anos neste cenário, tendo foco em quais são de fácil acesso e custo acessível.

\subsection{Software de interpretação estudado: Assistive Context-Aware Toolkit (ACAT)}

É um dos softwares testados que mostrou ter grande utilidade para o desenvolvimento dos protótipos propostos, pela integração que pode ser realizada.

Desenvolvido pela Intel Labs, é um plataforma de código aberto que tem como intuito permitir que pessoas com doenças neuromotoras e outras deficiências tenham acesso total às capacidades e aplicações de seus computadores por meio de interfaces muito restritas, adequadas à sua condição. Mais especificamente, o Assistive Context-Aware Toolkit [Intel 2019] permite que os usuários se comuniquem facilmente com outras pessoas por meio da simulação de um teclado, previsão de palavras e síntese de fala. Os usuários podem executar uma série de tarefas, como editar, gerenciar documentos, navegar na Web e acessar e-mails.

O ACAT é projetado para uso em máquinas Microsoft Windows do qual é possível fazer interface com diferentes entradas de sensores, como interruptores infravermelhos, câmera, botões e muito mais, sendo este recurso aproveitado neste projeto. Assim, procurou-se basear o desenvolvimento do protótipo de forma a realizar uma integração com o software.

\section{Procedimentos metodológicos}

\subsection{Caracterização do problema}

Para o desenvolvimento dos protótipos, fez-se necessário o estudo de um caso específico, que neste projeto foi um voluntário adulto possuidor de problemas motores decorrentes de paralisia cerebral. Quanto às suas limitações, ele consegue realizar movimentos com os membros superiores, porém, de maneira muito limitada. Basicamente, o voluntário consegue se movimentar, porém seus movimentos não possuem refinamento ou precisão em nenhum membro ou pescoço. Sua paralisia também dificulta a fala, o que descarta softwares de reconhecimento de voz.

Abaixo são brevemente descritos os protótipos e módulo de treinamento desenvolvidos, dada a condição do participante, unida com base no funcionamento do software de interpretação dos comandos (o ACAT), seguidos da descrição das tecnologias utilizadas, em sequência cronológica de seu desenvolvimento.

\subsection{Protótipo 1: Botão de tamanho aumentado}

Para a construção do primeiro protótipo, utilizou-se a funcionalidade disponível no software interpretador quanto à interação com o usuário através de uma tecla do computador, 
tendo como ideia inicial o desenvolvimento de um botão de tamanho aumentado com conexão USB, que estivesse mais próximo do usuário, a fim de tornar mais fácil de apertá-lo, comparado a uma tecla do teclado comum do computador ou notebook.

Com uma estética baseada em um gadget já comercializado no mercado, chamado "botão anti-estresse", o seu funcionamento ocorre por meio de um módulo de teclado que utiliza uma rede matricial que identifica cada tecla, graças a uma linha e uma coluna. A fim de seguir a proposta da criação de um dispositivo de baixo custo, os materiais providos para a construção do módulo de teclado são reciclados, do qual fez-se o reaproveitamento do módulo de um teclado para descarte, contribuindo para a redução de lixo eletrônico.

\subsection{Protótipo 2: Detecção de movimentos}

O segundo protótipo foi desenvolvido tendo como ideia-base o trabalho desenvolvido por [Pitaluga 2016], que trata-se de um painel de botões programados por Arduino funcionando como um teclado, adaptando-o para a detecção do movimento do usuário do dispositivo.

Aproveitou-se a mesma funcionalidade vista no protótipo anterior, ou seja, a interação com o ACAT apenas com o clique de um botão. Assim, desenvolveu-se um protótipo que envia o sinal de um botão ao software a partir da detecção de movimento.

Utilizar um sistema embarcado como o Arduino trouxe benefícios ao projeto, pelo fato de possibilitar utilizar tecnologias que captem informações de ambiente externo - no caso deste trabalho, detecção de movimento - como a ultrassônica, através de sensores de baixo custo, além de possuir uma gama de bibliotecas disponíveis via open source, como por exemplo a biblioteca Keyboard [Arduino 2015] utilizada no protótipo, a qual interpreta o sinal enviado para o microprocessador como um comando de teclado passado para o computador.

\subsection{Módulo de treinamento de utilização do Assistive Context-Aware Toolkit - (ACAT)}

Com base na interação do usuário alvo com o ACAT ao decorrer das fases de testes, observou-se uma complexidade no aprendizado do software, o que gerou a necessidade de se desenvolver também um treinamento em língua portuguesa para seu melhor uso, em paralelo aos protótipos desenvolvidos.

Ele é composto por módulos, por exemplo, "Como instalar o ACAT" (resumo sobre o aplicativo e suas aplicações e interações), "uso do Qwerty" (Primeiros passos com o app ACAT Tryout e ajuste de velocidade, apresentação do QWERTY e suas funções), entre outros.

Tal módulo, que tem como formato um vídeo descritivo e um texto base para auxílio, foi desenvolvido em paralelo ao presente trabalho e publicado na plataforma Medium. [Aires et al. 2018]

\section{Resultados e análise de desempenho}

A seguir serão apresentados os resultados obtidos no decorrer da construção de cada um dos protótipos desenvolvidos, assim como seus respectivos desempenhos ao serem testados. 
A fim de possuir um feedback mais assertivo quanto à funcionalidade de cada um, ao longo deste trabalho teve-se a participação de um voluntário adulto possuidor de deficiência motora acentuada e de fala, já descrita anteriormente, que se disponibilizou a testá-los, passando então uma ideia concreta dos acertos e erros cometidos durante seus desenvolvimentos.

\subsection{Teste do primeiro protótipo: Botão de tamanho aumentado}

Dada a proposta de um dispositivo de baixo custo, o botão de tamanho aumentado foi o protótipo com menor custo para construção, pelo uso de materiais recicláveis e de uso comum. A seguir, a Figura 1 apresenta, da esquerda para a direita respectivamente, o módulo de teclado reutilizado, o botão de acionamento protegido por papelão e o produto final, preenchido de espuma para aumentar a superfície de contato com o botão e melhorar o conforto no acionamento.
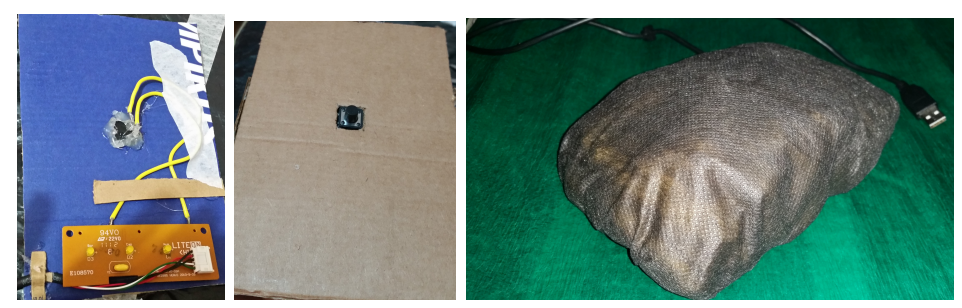

Figura 1. Etapas de construção do botão de tamanho aumentado

Ao entrar na fase de testes sua performance não se saiu satisfatória, ocasionada pela dificuldade vista em seu uso pelo participante que, por possuir alguns movimentos bruscos nos membros superiores, necessitou muitas vezes usar a força para acioná-lo, o que causou um grande desconforto quanto a usabilidade. Embora o voluntário não tenha se adaptado, o protótipo pode ser útil para pessoas com deficiência menos severa nos membros superiores.

\subsection{Teste do segundo protótipo: detector de movimento}

Nesta fase, já era de conhecimento que o uso de sensores poderia desempenhar melhores resultados comparados ao anterior, por exigirem o mínimo de esforço possível para a detecção de algum movimento realizado, apesar do seu custo ser consideravelmente maior - aproximadamente $\mathrm{R} \$ 60,00$. Contudo, este se mostrou um valor expressivamente mais acessível que a média dos dispositivos comerciais voltados à tecnologia assistiva.

Nos momentos iniciais de teste com o voluntário, este apresentou algumas dificuldades quanto ao seu uso, devido às limitações que possui nos movimentos realizados pelos membros superiores. Porém, foi possível melhorar a experiência com um simples reposicionamento do protótipo, tal que conseguisse captar gestos realizados pela inclinação do pescoço conjuntamente com o tronco, detectando seu queixo. Este é um movimento ao qual já estava familiarizado, por realizá-lo com frequência com sua ponteira física de teclado. Assim, uma vantagem do uso deste sensor foi sua flexibilidade, pois permitiu que o usuário realizasse o movimento que tinha maior facilidade de execução. A seguir, a Figura 2 apresenta, da esquerda para a direita respectivamente, o Arduino Leonardo e o sensor ultrassônico HC-SR04 utilizados, a disposição interna dos componentes na caixa de proteção e a construção final do protótipo 2. 


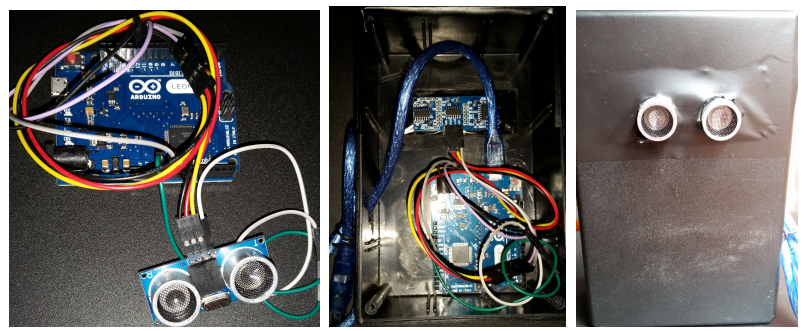

Figura 2. Etapas para construção do dispositivo usando sensor ultrassônico

\section{Considerações Finais}

Ao longo da execução do projeto foi possível perceber uma evolução na usabilidade dos dispositivos, dos quais a melhor opção foi o sensor ultrassônico para captação dos movimentos. Além disso, percebeu-se que a flexibilidade de posicionamento do sensor obteve grande importância para uma interação satisfatória.

Porém, apesar de todas as melhorias atingidas durante a execução do trabalho, percebeu-se que a complexidade de utilização do software interpretação, no caso o ACAT, não tornou o produto como um todo tão satisfatório quanto se esperava, mesmo com a tentativa da criação de um treinamento para utilizá-lo. Com base naquilo relatado pelo participante dos testes, a melhor opção seria um equipamento que fosse isento de um software auxiliar, o qual permitisse ao usuário ser mais independente, não precisando depender do processamento feito por um outro programa. Assim, como trabalho futuro, pretende-se dar continuidade a este projeto levando em consideração as propostas sugeridas pelo voluntário. Da mesma forma, pretende-se realizar testes de usabilidade com outros voluntários que possuam o mesmo tipo de deficiência.

\section{Referências}

Aires, M. C., Muniz, I. R. F., and Stringhini, D. (2018). Módulo de treinamento acat. Disponível em: <https://medium.com/@matheus.c.a66/mㄷ 3\% B3dulo-de-treinamento-acat-dce 50 f949d94 >.Acesso em: abr 2019.

Arduino (2015). Documentação de referência do arduino - biblioteca keyboard. Disponível em: <https://www.arduino.cc/reference/pt/language/ functions/usb/keyboard/>.Acesso em: nov. 2018.

Bersch, R. (2013). Introdução à Tecnologia Assistiva. Porto Alegre, RS.

Intel (2019). Assistive context-aware toolkit. Disponível em: <https://01 .org/ acat >.Acesso em: mar. 2019.

Macedo, P. C. M. (2008). Deficiência física congênita e saúde mental. Rev. SBPH, 11(2):127-139.

MacLennan, A. H., Thompson, S. C., and Gecz, J. (2015). Cerebral palsy: causes, pathways, and the role of genetic variants. American Journal of Obstetrics Gynecology, 213(6):779 - 788 .

Pitaluga, P. (2016). Arduino programmable button panel as keyboard. Disponível em: <https://www.instructables.com/id/ Arduino-Programmable-Button-Panel-As-Keyboard/>.Acesso em: nov. 2018. 